\title{
SEGMENTATION OF UNHEALTHY REGION OF PLANT LEAF USING IMAGE PROCESSING TECHNIQUES: A SURVEY
}

\author{
P.Kavitha ${ }^{1}$, B.Ananthi ${ }^{2}$ \\ ${ }^{1}$ Assistant Professor, Department Of Computer Applications, Erode Arts and Science College, Erode, Tamilnadu, \\ India \\ ${ }^{2}$ Assistant Professor, Department Of Computer Science (Ug \& Pg), Vellalar College for Women, Erode, Tamilnadu, \\ India
}

\begin{abstract}
A segmentation technique is used to segment the diseased portion of a leaf. Based on the segmented area texture and color feature, disease can be identified by classification technique. There are many segmentation techniques such as Edge detection, Thresholding, K-Means clustering, Fuzzy C-Means clustering, Penalized Fuzzy C-Means, Unsupervised segmentation. Segmentation of diseased area of a plant leaf is the first step in disease detection and identification which plays crucial role in agriculture research. This paper provides different segmentation techniques that are used to segment diseased leaf of a plant.
\end{abstract}

Keywords: Fuzzy C-Means, K-Means, Penalized FCM, Unsupervised Fuzzy Clustering

$* * *$

\section{INTRODUCTION}

Plant exists everywhere we live. India is an agriculture country where $70 \%$ (Pramod S.Landge et al) of population depends on agriculture. The cultivation of land requires close and continuous monitoring for the management of plant leaf disease that can affect production significantly. In practice disease of an plant leaf is identified by naked eye observation of an expert, which is too expensive and time consuming to make experts arrive to field. Instead ,in automatic detection and identification of plant leaf disease image of a leaf is captured and then segmented using segmentation techniques to extract infected area. In literature survey it presents some of those existing system.

\section{LITERATURE SURVEY}

Dheeb Al Bashidh \& et al [1] in the paper titled "Detection and Classification Of leaf Disease Using K-Means based Segmentation and Neural networks based classification" proposed an approach which consists of four main phases for five types of leaf disease. First color transformation structure for the RGB leaf image and then device independent color space transformation is applied, Next in second phase image is segmented using K-Means clustering technique, Thirdly calculate the texture feature of segmented area of leaf. Finally classification is done through pretrained neural network. K-Means clustering technique provides efficient results in Segmentation of RGB image. By KMeans segmentation multiple values of cluster have been tested. Best result were observed when the number of clusters is four.

Mohammed El-Hellyt \& et al [2] describe about Fuzzy CMean FCM clustering algorithm in the paper titled "Integrating Diagnostic expert system with Image Processing" to segment infected part of a leaf after image enhancement. From the segmented area features are extracted and classification is done using Artificial Neural Network. In this system they tested for 3 cucumber disorder.The result of this indicate that this system could identify disorder with high level of accuracy.

"Image segmentation by Fuzzy C-Means clustering algorithm with a Novel Penalty term " by Yony Yang [3] describe Fuzzy C-Means(FCM) which takes into account the influence of neighborhood pixels on the central axis .So the Penalized FCM algorithm includes the spatial information which is less sensitive to noise than FCM.

Jaingsheng Guis and et al [4] developed “ Unsupervised segmentation method for disease of soyabean color image based Fuzzy clustering "which gradually increase the number of cluster in FCM ,iteratively, in the process of each iteration apply validation criteria to evaluate the effectiveness of clustering. By this method optimal number of clusters and centers are obtained.

In the paper titled "Detection of unhealthy region of plant leaves and classification of plant leaf disease using texture features" by S.Arivazhagan and et al [5] segmented diseased portion of a leaf after masking green pixels and removing green portion of a leaf.The infected region is then segmented by applying specific threshold. Classification is done by Minimum distance classifier and support vector machine

This algorithm was testd on ten species of plants.

"An Empirical Investigation of olive Leave Spot Disease Using Auto-Cropping Segmentation and Fuzzy C-Means Classification" by Mokheld Al-Tarawneh [6] describe describe that FCM with polygon auto-cropping segmentation provides accuracy than K-Means segmentation. 
Hrushikesh Dattatray Marathe et al [7] presented a paper titled "Leaf Disease Detection Using Image Processing Techniques" describe detection of diseased portion of a leaf by subtracting total green area from total leaf area. This system implemented in MATLAB which makes calculation easy to extract the infected area.

\section{SEGMENTATION TECHNIQUES}

Segmentation is the process of partitioning a digital image into multiple regions or objects and extracting meaningful region known as the region of interest(ROI). The level to which subdivision is carried depends on the problem being solved. Segmentation can be stopped when the region of interest in an application have been isolated. Segmentation accuracy determines the eventual success or failure of computerized analysis procedures. So care should be taken to pick an algorithm that performs the best for the given requirement.

Image segmentation algorithms generally based on two basic principles: discontinuity and similarity .The discontinuity principle is to extract regions that differ in properties such as intensity, color, texture or any other image statistics. .In similarity principle an image pixels are grouped into regions that are similar according to a set of predefined criteria. Thresholding, region growing and region splitting and merging are example of similarity principle.

The segmentation algorithms can be classified on the basis of the pixel similarity relationship with neighbouring pixel as (i) Contextual (region-based or global) algorithms (ii)Non-contextual(pixel-based or local)algorithms.

In region-based algorithms, the pixels are grouped based on some sort of similarity that exists between them. In pixelbased the idea is to identify the discontinuities that are present in the image such as isolated lines and edges.

In plant leaf disease identification the following steps are followed.

1. Image is converted from RGB to HSI or gray scale image.

2. The infected areas of leaf are extracted by segmentation technique.

3. From extracted part, texture features of a region are calculated and disease is identified by using classification technique.

\section{CLUSTERING}

Clustering is a technique for partitioning a group of images into disjoint subgroups. Images that are similar to each other group themselves into a single cluster. All the images in subgroup are similar to each other. At the same time, the images across the cluster are different, In image segmentation, clustering is used to provide the updated centriod value based on the distance between the objects.

This section will describe some of the clustering techniques that are used to segment the infected area. The clustering techniques considered are K-Means, FCM, Penalized FCM and Unsupervised fuzzy clustering.

\subsection{K-Means Clustering}

K-Means is an hard clustering algorithm approach where the physical boundaries of cluster are well defined. In K-Means clustering algorithm the $\mathrm{n}$ objects or instances $x 1, x 2, x 3 \ldots x n$ is classified into $\mathrm{K}$ clusters with initial centroids.

The K-Means objective function is

$$
\mathrm{J}=\sum_{j=1}^{k} \sum_{i=1}^{n}\left\|x_{\mathrm{i}}-\mathrm{C}_{\mathrm{j}}\right\|
$$

$\mathrm{K}$ is the number of clusters.

$\mathrm{C}$ is the cluster centriod and $\mathrm{x}$ is the object.

Procedure for K-Means technique is

1. Intialize the centriod.

2. Find the distance between object and centeroid

3. Assign the object to the cluster with which distance is minimum

4. Recalculate the new mean of the cluster.

5. Repeat the step till a predefined threshold is met.

\section{Advantage:}

Segmentation of an image is fast for image where the boundaries are well defined.

\section{Disadvantage:}

The performance of the K-means algorithm depends on the initial positions of the cluster centers. This is an inherently iterative algorithm. And also there is no guarantee about the convergence towards an optimum solution. The convergence centroids vary with different initial points.

\subsection{Fuzzy C-Means Clustering}

Fuzzy logic deals with the vagueness and imprecision present in the problem. The image in grey color has an ambiguity in brightness and darkness of a pixel. In segmentation problem, the image is first converted from RGB to HIS which has confusion in determining whether pixel is normal or infected.This kind of vagueness is known as spatial ambiguity.

To solve this, image is considered as a fuzzy set. Fuzzy clustering obtain more reasonable results for vague cluster boundaries. In a fuzzy set there is a degree of membership for every member. Value of membership lies between 0 and 1 and the sum of membership of each object is 1.Larger the membership values indicates higher confidence to cluster.

Fuzzy C- Means objective function is

$$
J=\sum_{i=1}^{c} \sum_{j=1}^{N} U_{\mathrm{ij}}{ }^{\mathrm{m}}\left|X_{\mathrm{i}}-\mathrm{C}_{\mathrm{j}}\right| 1 \leq \mathrm{m} \leq \alpha
$$

Where $m$ is any real number greater than 1 , $c$ is number of clusters, 
$U_{i j}$ is the degree of membership of $x_{i}$ in the cluster $j$,

$U_{i j}$ is the degree of membership of $x_{i}$ in the cluster $j$,

$\mathrm{X}_{\mathrm{i}}-\mathrm{C}_{\mathrm{j}}$ is euclidean distance between any data object and the centroid of $\mathrm{j}^{\text {th }}$ cluster

Fuzzy C- means clustering algorithm is

1. Choose a number of clusters.

2. Assign randomly to each point in the coefficients for being in the clusters.

3. Repeat until the algorithm has convergence.

4. Compute the centriod for each cluster.

5. For each point compute its co-efficient in the cluster.

\section{Advantage}

1. In Fuzzy C-Means clustering each element is assigned to multiple cluster with certain degree of membership in the dataset. So it minimize intra-cluster variance.

2. FCM obtain more reasonable results where the boundaries are vague.

\section{Disadvantage}

1. Dependency on initial centriod.

2. Sensitivity to noise and outliners.

3. Results depend on initial centriod.

\subsection{Penalized Fuzzy C-Means}

The FCM clustering technique does not include any spatial information into the objects. By minimizing the objective function of neighborhood EM algorithm, Penalized FCM include space information and spatial information during segmentation which forms the new objective function.

The objective function of PFCM is

\section{$\mathrm{PFCM}=\mathrm{FCM}+\mathrm{NEM}$}

$$
\begin{aligned}
& \text { (i.e) } \mathbf{J}_{\mathrm{PFCM}}=\sum_{k=1}^{n} \sum_{i=1}^{c}(U \mathrm{ik})^{\mathrm{q}} \mathrm{d}^{2}\left(\mathrm{x}_{\mathrm{k}}, \mathrm{v}_{\mathrm{i}}\right)+ \\
& \quad \mathrm{r} \sum_{k=1}^{n} \sum_{j=1}^{n} \sum_{i=1}^{c}\left(u_{\mathrm{ik}}\right)^{\mathrm{q}}\left(1-\mathrm{u}_{\mathrm{ij}}\right)^{\mathrm{q}} \mathrm{w}_{\mathrm{kj}}
\end{aligned}
$$

Where

$\mathrm{w}_{\mathrm{kj}}=\left\{\begin{array}{c}1 \text { if } \mathrm{xj} \text { and } \mathrm{xk} \text { are neighbours and } \mathrm{j} \neq \mathrm{k} . \\ 0 \text { otherwise }\end{array}\right.$

\subsection{Image Processing Method}

In this method total leaf area is calculated through pixel number statistics. Next the green area of leaf is calculated .By subtracting green leaf area from total leaf area gives the infected area of the leaf. Its easy to calculate the leaf area by counting total pixel in a leaf area region.

\subsection{Unsupervised Fuzzy Clustering Algorithm}

Unsupervised Fuzzy clustering is a clustering based on FCM algorithm. Without knowing the number of clusters, by using fuzzy super volume and separation density function the effectiveness of clustering can be assessed effectively and can automatically obtain optimize number of clusters $\mathrm{c}$ and cluster centers. Here gradually increase the number of clustering ,iteratively, in the process of each iteration validation criteria is applied to evaluate the effectiveness of clustering.

\section{Algorithm:}

1. Select initial clusters, then set contrast coefficient $\mathrm{m}$, maximum allowable error $\mathrm{e}$ and maximum number of cluster $\mathrm{c}$.

2. For the cluster selected above FCM is applied to find initial cluster and Euclidean distance is used as a distance measure.

3. The distance measure is changed into exponential distance function using FCM clustering algorithm.

4. The effectiveness of clustering is calculated based on some parameters like hypervolume standard,classification density and average density of separation.

5. If this cluster is less than a predetermined maximum number of cluster ,then increase the number of cluster by 1 and repeat from step two.If it not so stop the calculation and apply the effective criteria of step 4 to the number of cluster.

\section{CONCLUSION}

This paper presents survey on different segmentation techniques that is used to segment the diseased part of a leaf.From the above segmentation techniques it is concluded that K-Means clustering works well if the boundaries are well defined .If the boundaries of an image is vague, FCM segmentation process produces good result. In penalized

FCM as it include neighborhood information which makes the segmentation easy than FCM. In simple image processing, the method is simple but as the pixel number is considered it discard pixel intensity into calculation. In unsupervised Fuzzy clustering it minimizes the number of iteration than FCM in finding convergence. But in unsupervised fuzzy clustering its complex in finding average density ,classification density and volume of a cluster.

\section{REFERENCES}

[1] Dheeb Al Bashidh, Mailk Braik and Sulieman Bani Ahmad 2011 "Detection and classification of leaf Disease using K-Means based segmentation and neural network classification," Information technology journal 10(2):267-275.

[2] Mohammed El-Helly “ Integrating Diagnostic expert system with Image processing" Central Lab. for agricultural research center.

[3] Yong yang and shuying Huang 2007 “ Image segmentation by Fuzzy C-Means clustering algorithm with a novel penalty term",Computing and Informatics, vol 26,17-37.

[4] Jaingsheng Gui,Li Hao,Shusen Sen,Wenshu Li,Yanfei Liu 2013 ,"Unsupervised Segmentation Method for Disease Of Soyabean Color Image based on Fuzzy Clustering"Sensors and Transducers vol 159,32-38.

[5] S.Arivazhagan, R.Newlin Shebiah, S.Ananthi, S.Vishnu varthini 2013,"Detection of unhealthy 
region of plant leaves and classification of plant disease using texture features" Agricultural engineering Int:CIGR Journal vol 15,211

[6] Mokheld Al-Tarawneh 2013 "An empirical investigation of Olive leaf spot disease Using Auto -Cropping Segmentation and Fuzzy C-Means Classification" World Applied Science journal 23(9);1207-1211

[7] Hrushikesh Dattatray Marathe and Prerna Namdeorao Kothe 2013 "Leaf Disease Detection Using Image Processing Technique" vol 2,ISSN:2278-0181.

[8] "Digital Image Processing “ by Rafael C.Gonzalez,Richard E.Woods

[9] "Digital Image Processing " by Sridhar. 